\title{
Risk Assessment of Retrograde Intramedullary Nailing for Proximal Humeral Fracture
}

\author{
Rina Sakai $^{1}{ }^{\circledR}$, Uchino Masataka², Kazuhiro Yoshida1 ${ }^{1}$, Masanobu Ujihira $^{1}$ \\ ${ }^{1}$ Department of Biomedical Engineering and Technology, School of Allied Health Sciences, Kitasato University, \\ Sagamihara, Japan; ${ }^{2}$ Department of Orthopedic Surgery, Hakujikai Memorial General Hospital, Tokyo, Japan \\ Correspondence to: Rina Sakai, rinax@kitasato-u.ac.jp
}

Keywords: Proximal Humeral Fracture, Retrograde Intramedullary Nailing, Anterograde Intramedullary Nailing, Locking Plate, Internal Fixation

Received: March 26, 2019 Accepted: May 18, 2019 Published: May 21, 2019

Copyright $\odot 2019$ by authors and Scientific Research Publishing Inc.

This work is licensed under the Creative Commons Attribution International License (CC BY 4.0).

http://creativecommons.org/licenses/by/4.0/

\section{(c) (i) Open Access}

\section{ABSTRACT}

In proximal humeral fracture except $\mathrm{AO}$ classification $11 \mathrm{~A} 1$, fixations with a locking plate and nails are recommended. We performed mechanical tests to investigate whether retrograde intramedullary nailing has fixation stability comparable to those of anterograde intramedullary nailing and locking plate which achieve clinically favorable outcomes. In retrograde intramedullary nailing, a nail entry point is made in the diaphysis, for which reduction of stiffness of the humerus is of concern. Thus, we investigated the influence of a nail entry point made in the diaphysis on humeral strength. Retrograde intramedullary nailing had fixation stability against bending and a force loaded in the rotation direction comparable to those of anterograde intramedullary nail and locking plate. Displacement by the main external force loaded on the humerus, compressive load, was less than half in the bone fixed by retrograde intramedullary nailing compared with that in the bone fixed with a locking plate, showing favorable fixation stability. It was clarified that stiffness of the humerus against rotation and a load in the compression direction is not reduced by a nail entry point made by retrograde intramedullary nailing.

\section{INTRODUCTION}

Fracture collapses continuity of the bone and results in morbid deformation, loss of supporting function, and pain. The bone function can be restored and pain is reduced by surgical stabilization of fracture. The final goal of internal fixation of fracture is to completely restore the bone function as quickly as possible. Reliable union is just one of the factors of functional recovery, but biomechanical studies on internal fixation methods are essential to achieve favorable outcomes [1]. 
The incidence of AO classification 11A2.1 proximal humeral fracture is high in the elderly. According to a large-scale non-randomized observational study, reduction of the function and non-union were noted in elderly patients with conservatively treated AO classification 11A2.1 fracture [2], whereas patients treated with internal fixation returned to daily life. For internal fixation of almost all of proximal humeral fracture, fixations with a locking plate and nails are recommended [3].

Locking plates are widely used clinically and superior outcomes in biomechanical studies have been occasionally reported $[4,5]$. However, locking plate fixation requires a large skin incision, being not low-invasive. Moreover, varus deformation, damage of the axillary nerve, and loss of blood supply to the humeral head are of concern $[6,7]$.

It has been reported that stiffness of bone fixed with anterograde intramedullary nailing is higher than that of bone fixed with a locking plate $[8,9]$. However, antegrade intramedullary nailing requires cutting the rotator cuff. The rotator cuff is a collection of tendons of four muscles: the subscapularis muscle, supraspinatus muscle, infraspinatus muscle, and teres minor muscle, and it is involved in elevation of the upper arm. Since shoulder joint contracture readily occurs, initiation of rehabilitation early after surgery is important, for which it is desirable not to cut the rotator cuff involved in elevation of the upper arm.

Retrograde intramedullary nailing was developed to avoid these problems. In this study, mechanical tests were performed to investigate whether the fixation stability of retrograde intramedullary nailing is comparable to those of antegrade intramedullary nailing and locking plate. Since a nail entry point is made in the diaphysis in retrograde intramedullary nailing, reduction of stiffness of the humerus is of concern. Thus, the influence of a nail entry point made in the diaphysis on humeral strength was investigated.

\section{MATERIALS AND METHODS}

\subsection{Evaluation of Fixation}

Two-part fracture with a $5 \mathrm{~mm}$ gap in the fracture region was prepared by applying AO classification 11A2.1 osteotomy to artificial left humerus (1013, Sawbones, USA, WA). The test implants were proximal retrograde humeral nails (MIZUHO, Japan), antegrade intramedullary nails (Trigen Proximal Straight Nail, Smith \& Nephew, UK), and a locking plate (PHILOS Plate, DePuy Synthes, USA, MA) (Figure 1). The three types of internal fixation were applied following the standardized surgical procedures. To evaluate fixation, bending, torque, and compression tests were performed.
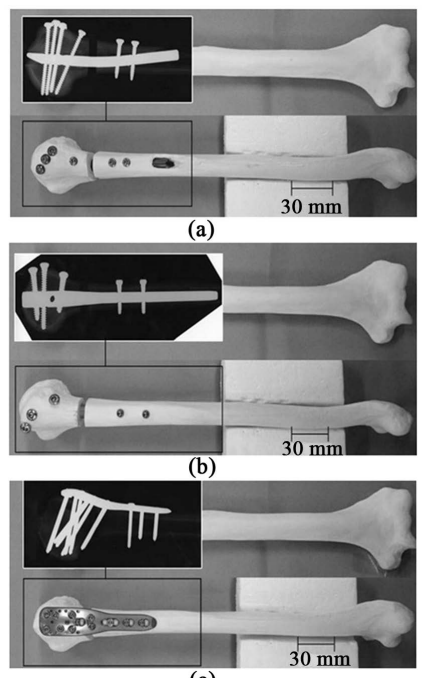

(c)

Figure 1. Internal fixation and AO classification 11A2.1 models of humeral fracture. Top: X-ray image of internal fixation. Bottom: Internal fixation and AO classification 11A2.1 model. (a) Retrograde intramedullary nailing; (b) Anterograde intramedullary nailing; (c) Locking plate. 
For the bending test, FGS-50VB-L (NIDEC, Japan) was used. The distal end of the humerus was fixed to a jig and the bone was placed horizontally setting the fulcrum at $45 \mathrm{~mm}$ from the distal end. A force was loaded on the humeral head at $20 \mathrm{~mm}$ proximal to the fracture region at $60 \mathrm{~mm} / \mathrm{min}$ until the force reached $50 \mathrm{~N}$ and the displacement angle was measured.

For the torque test, an electric torque stand, DSP-10 (NIDEC, Japan), and digital torque meter, TNX-10 (NIDEC, Japan), were used. The proximal end of the humerus was $8^{\circ}$ rotated at a speed of 0.2 $\mathrm{rpm}$ to reproduce a torque load on the humerus, and the torque generated during rotation was measured.

For the compression test, a loading test machine, FGS-50VB-L (NIDEC, Japan), was used for measurement, and the force loaded was controlled using a digital force gauge, FGP-100 (NIDEC, Japan). Resin OSTRONII (GC, Japan) was crimped to the distal humerus and molded so as to stand the bone vertically upright. A detachable cap was used to avoid direct loading on the intramedullary nail. A force was loaded on the proximal end of the humerus and displacement of the fracture region was measured.

\subsection{Influence of a Nail Entry Point on Stiffness of the Humerus}

Artificial left humerus (1013, sawbones, USA, WA) with and without a nail entry point were prepared (Figure 2). Nail entry points were made using a guide pin, starting reamer, and counter sink used in the surgical procedure of retrograde intramedullary nailing. A burr hole with a $10 \mathrm{~mm}$ diameter was made using a drill at $100 \mathrm{~mm}$ from the humeral head on the lateral side of the diaphysis. The distal end was covered with resin OSTRONII (GC, Japan) to stably stand the bone upright in the torque and compression tests.

The humerus was fixed at $40 \mathrm{~mm}$ from the humeral head and placed on the jig setting the fulcrum at $50 \mathrm{~mm}$ from the fixation site and the nail entry point facing up horizontally. Using a loading test machine, FGS-50VB-L (NIDEC, Japan), each test fragment was subjected to the bending test. A force was loaded on the diaphysis $170 \mathrm{~mm}$ distal to the humeral head at $60 \mathrm{~mm} / \mathrm{min}$ until reaching $50 \mathrm{~N}$. The stiffness was calculated from the relationship between the load added and displacement angle.

Using a digital torque meter, TNX-10 (NIDEC, Japan), each test fragment was subjected to internal and external rotation tests. The distal end and humeral head of the test fragment were fixed with clamps. The humeral head was $8^{\circ}$ externally rotated at a torque speed of $1 \mathrm{rpm}$, and the maximum torque loaded on the distal end of the humerus was measured. The maximum torque was divided by the internal and external rotation angles and evaluated as stiffness.

The compression test was performed using a loading test machine, FGS-50VB-L (NIDEC, Japan). To apply a load homogenously on the humeral head, a detachable cap was prepared with resin and the cap was interposed in the proximal end of the humerus during the test. A force was loaded at $60 \mathrm{~mm} / \mathrm{min}$ until reaching $500 \mathrm{~N}$. The stiffness of each test fragment was calculated from the relationship between the load added and displacement.

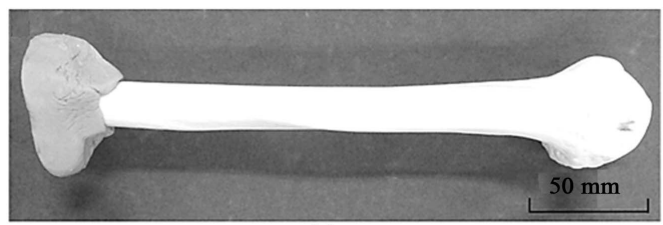

(a)

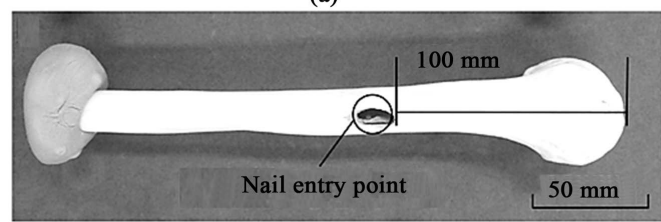

(b)

Figure 2. Specimens. (a) Proximal humerus without a nail entry point; (b) Proximal humerus with a nail entry point. 


\section{RESULTS}

The humeral diaphysis displacement angles were $12.20 \pm 3.19,9.71 \pm 2.34$, and $9.50 \pm 1.46$ degrees in the retrograde intramedullary nail, antegrade intramedullary nail, and locking plate groups, respectively, showing no significant difference (Figure 3 ). The torques generated at the distal end of the humerus were $0.88 \pm 0.07,0.79 \pm 0.17$, and $1.03 \pm 0.05 \mathrm{Nm}$, respectively, showing a significant difference between the antegrade intramedullary nail and locking plate groups (Figure 4). The gap displacements in the fracture region were $0.92 \pm 0.25,0.80 \pm 0.18$, and $2.30 \pm 0.27 \mathrm{~mm}$, respectively, showing a significant difference between the locking plate and each of the intramedullary nail groups (Figure 5).

The humeral stiffness calculated from the relationship between the load added and displacement in the bending test was $11.9 \pm 1.18$ and $16.4 \pm 0.87 \mathrm{~N} / \mathrm{mm}$ in the groups with and without a nail entry point, respectively, showing a significant difference between the two groups, and the stiffness was $25 \%$ lower in the presence of a nail entry point compared with that in the absence of a nail entry point (Figure 6(a)). On the torque test, rotation stiffness of the humerus was $0.14 \pm 0.02$ and $0.17 \pm 0.04 \mathrm{Nm} /$ degree in the groups with and without a nail entry point, respectively, showing no significant difference between the two groups, and the stiffness was $18 \%$ reduced in the presence of a nail entry point compared with that in the absence of a nail entry point (Figure 6(b)). On the compression test, the humeral stiffness calculated from the relationship between the load added and displacement was $243 \pm 30.0$ and $246 \pm 22.7 \mathrm{~N} / \mathrm{mm}$ in the groups with and without a nail entry point, respectively, showing no significant difference, and the stiffness was $1 \%$ reduced in the presence of a nail entry point compared with that in the absence of a nail entry point (Figure 6(c)).

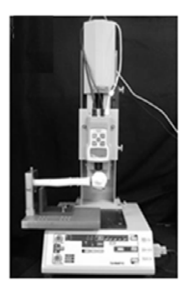

(a)

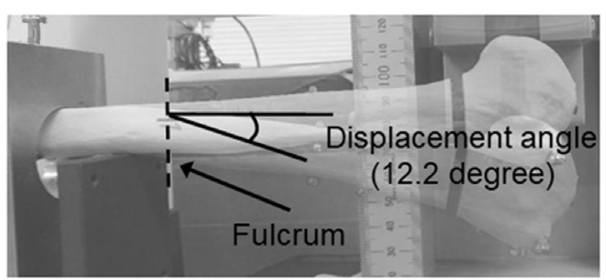

(b)

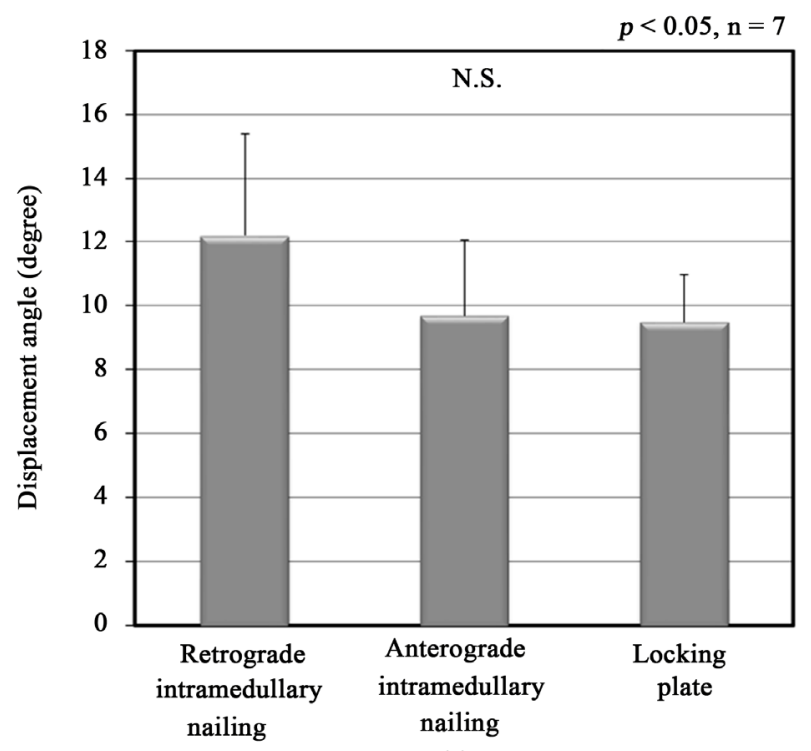

(c)

Figure 3. Bending testing. (a) Apparatus appearance of bending testing machine; (b) Measurement of displacement angle; (c) Displacement angle of internal fixations. 


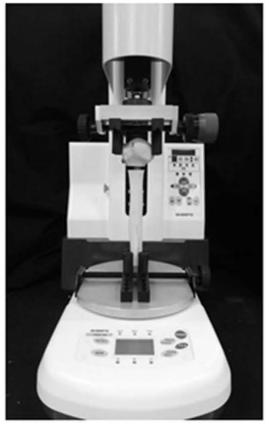

(a)

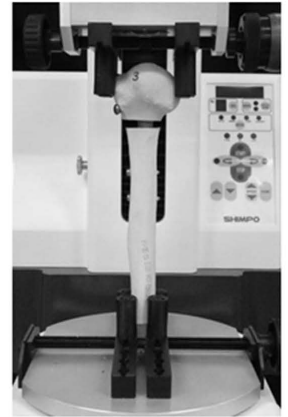

(b)

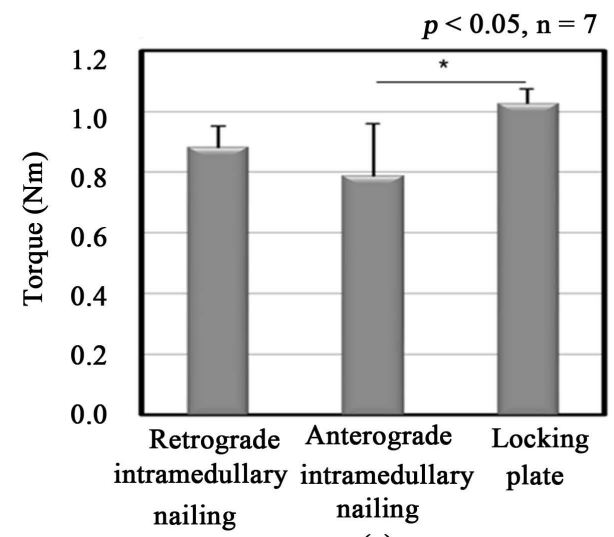

(c)

Figure 4. Torque testing. (a) Torque testing machine; (b) Enlarged measurement unit; (c) Torque of internal fixation.

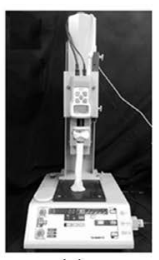

(a)

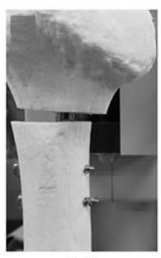

(b)

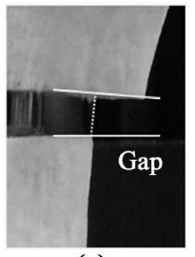

(c)

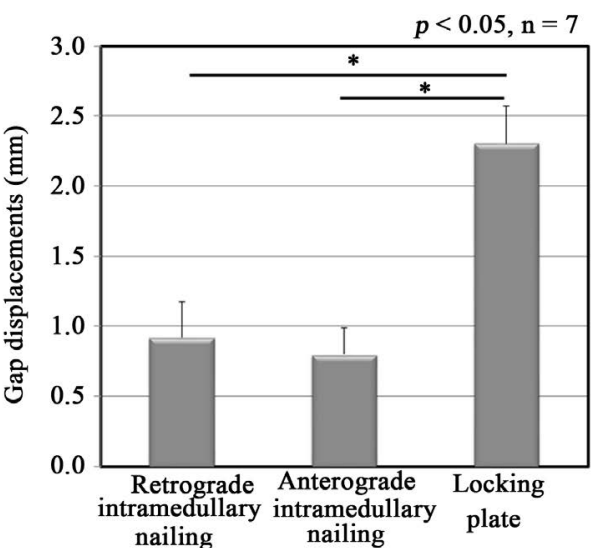

(d)

Figure 5. Compression testing. (a) Apparatus appearance of compression testing machine; (b) Enlarged measurement point; (c) Enlarged gap displacement; (d) Gap displacement of internal fixations. 


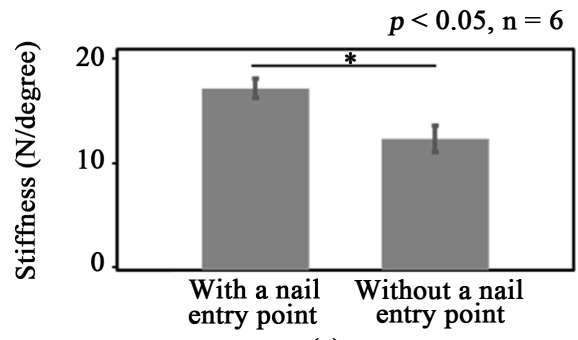

(a)

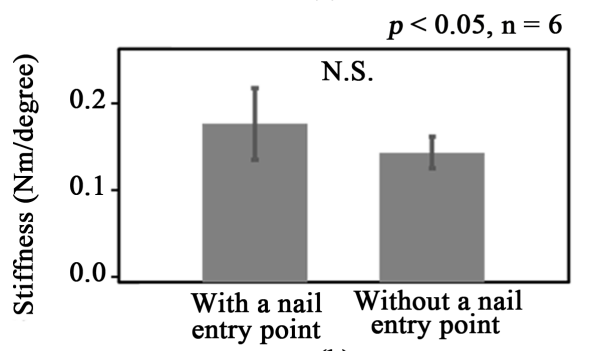

(b)

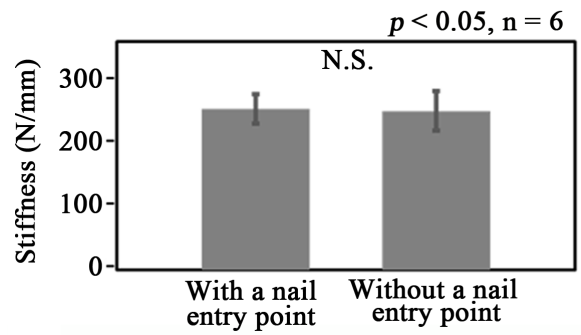

(c)

Figure 6. Stiffness of the proximal humerus with a nail entry point and without a nail entry point. (a) Bending testing; (b) Torque testing; (c) Compression testing.

\section{DISCUSSION}

A compressive load generated when one places a hand on the ground may be the most frequent external force loaded on the humerus. The gap displacements in the bones fixed with retrograde and antegrade intramedullary nails were less than half of that in the bone fixed with a locking plate, suggesting a favorable fixation ability of retrograde and antegrade intramedullary nailing. Since a locking plate supports the humerus on the lateral side, a varus moment may have been generated against the plate and increased displacement by compression. Since no significant difference was noted in the value of the bending or torque test in retrograde intramedullary nailing from those in the other internal fixations, retrograde intramedullary nailing can be judged as having a fixation ability comparable to those of the other internal fixations. Since damage of the axillary nerve, which is caused by plate fixation, can be avoided by this fixation method and the risk of damage of the rotator cuff, which may occur in antegrade intramedullary nailing, is small, retrograde intramedullary nailing is a useful internal fixation method for proximal humeral fracture with regard to reducing invasiveness [10].

The influence of a nail entry point was investigated. On the bending test, in which a significant difference was noted, stiffness decreased by $25 \%$, but about $20 \%$ reduction of bone stiffness may occur within a physiological range, such as that due to reduction of hormone [11]. The bending test may represent a state with deformation in the direction orthogonal to the axis caused by a force loaded to the cantilever. Thus, tensile stress may have been generated on the lateral side of the upper arm and stress may have been concentrated around the nail entry point, increasing the stiffness reduction rate. In daily life, a force in the bending direction may be loaded while lifting things. It is necessary to be careful when the upper arm is elevated while holding luggage. Opening and closing a pet bottle cap and turning a doorknob are forces 
loaded while rotating the arm around the bone axis in daily life, but these are unlikely to load a local force on a nail entry point. Getting the hand on the ground while standing up and falling is a motion loading a force in the bone axis direction. Considering that many patients are elderly, this is the most expected load. Since no influence of a nail entry point was noted in this loading, the fracture risk due to the use of retrograde intramedullary nailing may be small.

For rehabilitation after retrograde intramedullary nailing, range of motion training of the shoulder joint is started one day after surgery without limitation in the range of motion, but healing of the nail entry point requires several weeks. Physicians need to instruct patients to be careful about elevating the upper arm while holding luggage during this period. Retrograde intramedullary nailing does not damage the rotator cuff or articular cartilage. As the screw holes of the nails are threaded, back-out and mobility of screws can be reduced. In addition, insertion of all screws through the device is possible, enabling a shorter exposure time, which is advantageous for surgeons. Although the number of patients treated by retrograde intramedullary nailing was insufficient to evaluate the clinical outcome, this method may become a surgical treatment option because the clinical outcome was favorable and the fixing force was comparable to that of the conventional implant.

Regarding the limitation of this study, the values were acquired in the experimental environment and these do not secure the superiority or inferiority of the fixation methods in clinical cases. It has been reported that fracture of the proximal end of the humerus occurs in patients with vulnerability of bone [12], but physical properties of the resin-made models of the humerus used as the test fragments in this study were not comparable to those of bone, so that reproducibility is poor. Moreover, the influences of soft tissue and ligament around the humerus were not reflected. The results corresponding to clinical cases may be acquired by performing mechanical tests using cadavers and analysis on the assumption of soft tissue and ligament. Collection of basic data from the fracture region facilitates correct selection of an internal fixation method for the treatment of fracture.

\section{CONCLUSION}

In the humerus simulating AO classification 11A2.1, three types of internal fixation method were subjected to bending, torque, and compression tests. It was suggested that the fixation ability of retrograde intramedullary nailing is comparable to those of antegrade intramedullary nailing and locking plate. The influence of a nail entry point made in the diaphysis by retrograde intramedullary nailing was investigated. It was clarified that humeral stiffness against rotation and load in the compression direction does not decrease.

\section{CONFLICTS OF INTEREST}

The authors declare no conflicts of interest regarding the publication of this paper.

\section{REFERENCES}

1. Thomas, P.R., Christopher, G.M. and Richard, E.B. (2007) AO Principles of Fracture Management. George Thieme Verlag, 2 Har/Dvdr.

2. Court-Brown, C.M., Garg, A. and McQueen, M.M. (2001) The Translated Two-Part Fracture of the Proximal Humerus. Epidemiology and Outcome in the Older Patient. The Journal of Bone \& Joint Surgery (British Volume), 83, 799-804. https://doi.org/10.1302/0301-620x.83b6.0830799

3. Dietz, S.O., Hartmann, F., Schwarz, T., Nowak, T.E., Enders, A., Kuhn, S., Hofmann, A. and Rommens, P.M. (2012) Retrograde Nailing versus Locking Plate Osteosynthesis of Proximal Humeral Fractures: A Biomechanical Study. Journal of Shoulder and Elbow Surgery, 21, 618-624. https://doi.org/10.1016/j.jse.2011.04.013

4. Huff, L.R., Taylor, P.A., Jani, J., Owen, J.R., Wayne, J.S. and Boardman, N.D. (2013) Proximal Humeral Fracture Fixation: A Biomechanical Comparison of Two Constructs. Journal of Shoulder and Elbow Surgery, 22, 
129-136. https://doi.org/10.1016/j.jse.2012.01.003

5. Yamamoto, N., Hongo, M., Berglund, L.J. Sperling, J.W., Cofield, R.H., An, K.N. and Steinmann, S.P. (2013) Biomechanical Analysis of a Novel Locking Plate with Smooth Pegs versus a Conventional Locking Plate with Threaded Screws for Proximal Humerus Fractures. Journal of Shoulder and Elbow Surgery, 22, 445-450. https://doi.org/10.1016/j.jse.2012.04.005

6. Bae, J.H., Oh, J.K., Chon, C.S., Oh, C.W., Hwang, J.H. and Yoon, Y.C. (2011) The Biomechanical Performance of Locking Plate Fixation with Intramedullary Fibular Strut Graft Augmentation in the Treatment of Unstable Fractures of the Proximal Humerus. The Journal of Bone \& Joint Surgery (British Volume), 93, 937-941. https://doi.org/10.1302/0301-620x.93b7.26125

7. Zettl, R., Müller, T., Topp, T., Lewan, U., Krüger, A., Kühne, C. and Ruchholtz, S. (2011) Monoaxial versus Polyaxial Locking Systems: A Biomechanical Analysis of Different Locking Systems for the Fixation of Proximal Humeral Fractures. International Orthopaedics, 35, 1245-1250. https://doi.org/10.1007/s00264-011-1220-z

8. Horn, J., Gueorguiev, B., Brianza, S., Steen, H. and Schwieger, K. (2011) Biomechanical Evaluation of Two-Part Surgical Neck Fractures of the Humerus Fixed by an Angular Stable Locked Intramedullary Nail. Journal of Orthopaedic Trauma, 25, 406-413. https://doi.org/10.1097/bot.0b013e3181ffdf9a

9. Rothstock, S., Plecko, M., Kloub, M., Schiuma, D., Windolf, M. and Gueorguiev, B. (2012) Biomechanical Evaluation of Two Intramedullary Nailing Techniques with Different Locking Options in a Three-Part Fracture Proximal Humerus Model. Clinical Biomechanics, 27, 686-691. https://doi.org/10.1016/j.clinbiomech.2012.03.003

10. Uchino, M. and Sakai, R. (2018) Treatment of Retrograde Intramedullary Nailing for Proximal Humeral Fractures. Journal of Japanese Society for Fracture Repair, 40, 435-435.

11. Fujiwara, S., Fukunaga, M., Nakamura, T., Chen, J.T., Shiraki, M., Hashimoto, T., Yoh, K., Nakamura, T., Mizunuma, H., Tomomitsu, T., Kasagi, F., Masunarim, N. and Orimom, H. (1998) Rate of Change in Spinal Bone Density among Japanese Women. Calcified Tissue International, 63, 202-207. https://doi.org/10.1007/s002239900515

12. Edwards, S.L., Wilson, N.A., Zhang, L.Q., Flores, S. and Merk, B.R. (2006) Two-Part Surgical Neck Fractures of the Proximal Part of the Humerus. A Biomechanical Evaluation of Two Fixation Techniques. The Journal of Bone \& Joint Surgery (American Edition), 88, 2258-2264. https://doi.org/10.2106/00004623-200610000-00020 